\title{
TIMP-2 SNPs rs7342880 and rs4789936 are linked to risk of knee osteoarthritis in the Chinese Han Population
}

\author{
Pengcheng $\mathrm{Xu}^{1, *}$, Wen Guo ${ }^{1, *}$, Tianbo Jin ${ }^{2,3}$, Jihong Wang ${ }^{4}$, Dongsheng Fan ${ }^{4}$, \\ Zengtao Hao ${ }^{4}$, Shangfei Jing ${ }^{4}$, ChaoQian Han ${ }^{4}$, Jieli Du${ }^{1}$, Dong Jiang ${ }^{1}$, Shuzheng \\ Wen $^{4}$, Jianzhong Wang ${ }^{5}$ \\ ${ }^{1}$ Inner Mongolia Medical University, Hohhot 010010, Inner Mongolia, China \\ ${ }^{2}$ Key Laboratory of Resource Biology and Biotechnology in Western China (Northwest University), Ministry of Education, \\ School of Life Sciences, Northwest University, Xi'an, Shaanxi 710069, China \\ ${ }^{3}$ Xi'an Tiangen Precision Medical Institute, Xi'an, Shaanxi, 710075, China \\ ${ }^{4}$ Department of Hand and Foot Surgery, Second Affiliated Hospital, Inner Mongolia Medical University, Hohhot 010030, Inner \\ Mongolia Autonomous Region, China \\ ${ }^{5}$ Department of Trauma, Second Affiliated Hospital, Inner Mongolia Medical University, Hohhot 010030, Inner Mongolia \\ Autonomous Region, China \\ *These authors have contributed equally to this work \\ Correspondence to: Shuzheng Wen, email: wenshuzhengdoctor@163.com \\ Jianzhong Wang, email: wangjianzhong0503@126.com \\ Keywords: knee osteoarthritis, TIMP-2, single nucleotide polymorphism, gene \\ Received: July 11,2016 Accepted: November 09, $2016 \quad$ Published: November 25, 2016
}

\section{ABSTRACT}

This study aimed to investigate whether functional polymorphisms in the tissue inhibitors of metalloproteinase-2 (TIMP-2) gene are associated with susceptibility to knee osteoarthritis (OA) in the Chinese Han population. Six TIMP-2 single nucleotide polymorphisms (SNPs) were assayed using MassARRAY in 300 patients clinically and radiographically diagnosed with knee $O A$ and in 428 controls. Allelic and genotypic frequencies were compared between groups. Logistic regression adjusting for age and gender was used to estimate risk associations between specific genotypes and knee OA by computing odds ratios (ORs) and $95 \%$ confidence intervals ( $95 \%$ CIs). We found that allele " $A$ " in rs7342880 was significantly associated with increased risk of knee $O A(O R=1.44,95 \% C I=1.09-1.91, p=0.035)$. In addition, in the overdominant model, rs4789936 correlated with reduced risk of knee OA, adjusting for age and gender $(\mathrm{OR}=0.69,95 \% \mathrm{CI}=0.49-0.98, p=0.036)$. Finally, rs7342880 correlated with increased risk of knee OA in females. This study provides evidence that TIMP-2 is a knee OA susceptibility gene in the Chinese population and a potential diagnostic and preventive marker for the disease.

\section{INTRODUCTION}

Knee osteoarthritis $(\mathrm{OA})$ is the most common type of arthritis and a leading cause of chronic disability. It affects $10 \%$ of men and $18 \%$ of women over 60 years of age and leads to substantial cost to the individual and society [1-3]. The disease is characterized by morphological, biochemical, molecular, and biomechanical changes of both cells and extracellular matrix (ECM) which lead to a slowly progressive loss of articular cartilage, sclerosis of subchondral bone, osteophytes, and subchondral cysts [4]. Metalloproteinase family proteins (MMPs) control ECM integrity by catalyzing the degradation of structural proteins including laminin, fibronectin, and various types of collagen $[5,6]$. The degradation of structural proteins in cartilage, which is a feature of arthritic diseases, is accelerated by increased expression of MMPs by chondrocytes [7]. On the other hand, tissue inhibitors of metalloproteases (TIMPs) inhibit the activity of metalloproteinases by interacting with the MMP active site [8]. Therefore, osteoarthritic cartilage is characterized by an imbalance between MMPs and TIMPs. 
Although the etiology and pathogenesis of knee OA remain unclear, some factors that may increase the risk of knee OA are undisputed, such as gender, advanced age, ethnicity, behavioral influences, obesity, occupation, and previous injury [9-11]. Furthermore, genetic variation remains the strongest predictor of the onset and progression of this disease [12-15].

Bhumsuk Keam et al. [16] identified the SNP rs4789934 in the TIMP-2 gene as a potential susceptibility locus for knee OA $\left(p=4.01 \times 10^{-6}\right)$ in a GWAS of 3,793 samples (476 cases: wrist + knee and 3317 controls). JingBo Ji et al. [17] confirmed that the expression of serum TIMP-2 is significantly decreased in an animal model of OA. Fang-Jie Zhang et al. [18] carried out an in vitro experimental study using tissue samples from 16 Chinese patients with knee OA and identified mRNA expression of TIMP-1 and TIMP-2 significantly increased cytotoxicity or apoptosis of chondrocytes. Additionally, Yiqian Liang et al. [19] found no significant association for rs2277698 in TIMP-2 gene with knee OA in another case-control study involving a Korean population. To date, no studies have assessed the correlation between SNPs in TIMP-2 and the risk of knee OA in the Chinese Han population. In the present case-control study, we evaluated the effect of TIMP-2 polymorphisms on knee OA in the Chinese Han population, based on the findings of the GWAS using populations of Korean ancestry.

\section{RESULTS}

\section{Participant characteristics}

The demographic characteristics of the study population, including gender, age, and course of disease, are summarized in Table 1. The study included 300 OA cases (100 male and 200 female) and 428 controls (197 male and 231 female). The mean age of the patients and the control group were $52.71 \pm 8.762$ years (range: 47-72 years) and $60.64 \pm 4.822$ years (range: $41-75$ years), respectively. OA patients were divided into two groups based on the time course of their disease $(<10$ years $\&$ $\geq 10$ years), and there were significant differences between these groups in age $(p<0.001)$ and gender $(p=0.001)$.

\section{Association between TIMP-2 polymorphisms and knee OA risk}

The basic information related to candidate SNPs in our study such as chromosomal position, gene, allele, HWE test results, and minor allele frequency (MAF) appear in Table 2. We assumed that the minor allele of each SNP was a risk allele compared to the wild-type allele. We used $\chi^{2}$ test to compare the differences in frequency distributions of alleles between cases and controls and found just one significant SNP in the TIMP-2 gene at a 5\% level (allele " $\mathrm{A}$ " in rs7342880, $p=0.011$, OR
$=1.44,95 \% \mathrm{CI}=1.09-1.91)$. Rs2003241 was excluded for significant deviation from Hardy-Weinberg Equilibrium (HWE) $(p<0.05)$. The HWE in other SNPs in the control group was similar to those of the HapMap Asian population (http://hapmap.ncbi.nlm.nih.gov/).

The association results between TIMP-2 SNPs and risk of knee OA under the genetic model are listed in Table 3. Before adjustment, we identified the minor "A" allele in rs7342880 was associated with an increased risk of knee OA based on analysis using the co-dominant, dominant, and additive models ( $p<0.05$ for all) in Table 4. After adjustment for gender and age, the overdominant model showed that the rs4789936 SNP was significantly associated with a 0.69 -fold decreased OA risk $(p<0.05)$. Nevertheless, the genetic model in rs 7342880 showed no significant difference between OA patients and controls $(p>0.05)$ after adjustment. In our statistical analysis, we found no statistically significant associations between SNPs and risk of knee OA after Bonferroni correction. This may be due to the relatively small sample size or the weakness of Bonferroni correction itself (the interpretation of a finding depends on the number of other tests performed).

\section{Association between TIMP-2 polymorphisms and knee OA patient clinicopathological features}

We investigated the interactions between clinical characteristics and the SNPs. Gender was the only significant variable, and among females the genotype "CA+AA" in rs7342880 SNP was associated with 1.708fold increased odds of knee OA, compared to genotype $\mathrm{CC}$, adjusting for age and gender (adjusted $\mathrm{OR}=1.708, p$ $=0.048,95 \% \mathrm{CI}=1.004-2.903$ ) (Table 4).

\section{Haplotype analysis}

One block (rs2277698 and rs11654470) was detected in the TIMP-2 SNPs by haplotype analyses (Figure 1). The results of the association between the TIMP-2 haplotype and the risk of knee OA are listed in Table 5. In this block, haplotype "CCAT" was associated with risk of knee $\mathrm{OA}(\mathrm{OR}=1.39 ; 95 \% \mathrm{CI}=1.04-1.86 ; p=$ $0.025)$. However, when adjusted by gender and age, there was no association between this TIMP-2 haplotype and the risk of knee OA $(p>0.05)$.

\section{Stratified analysis}

In stratified analysis by gender, we found rs 7342880 SNP was not significantly associated to knee OA in males $(p>0.05)$ but was statistically significant in females $(p$ $<0.05$ ) (Table 6). We took common genotypes as the reference in male or female controls. The "CA+ AA" genotype of rs7342880 was associated with increased knee OA risk in females $(p=0.048, \mathrm{OR}=1.55,95 \% \mathrm{CI}$ 
Table 1: Characteristics of the study subjects

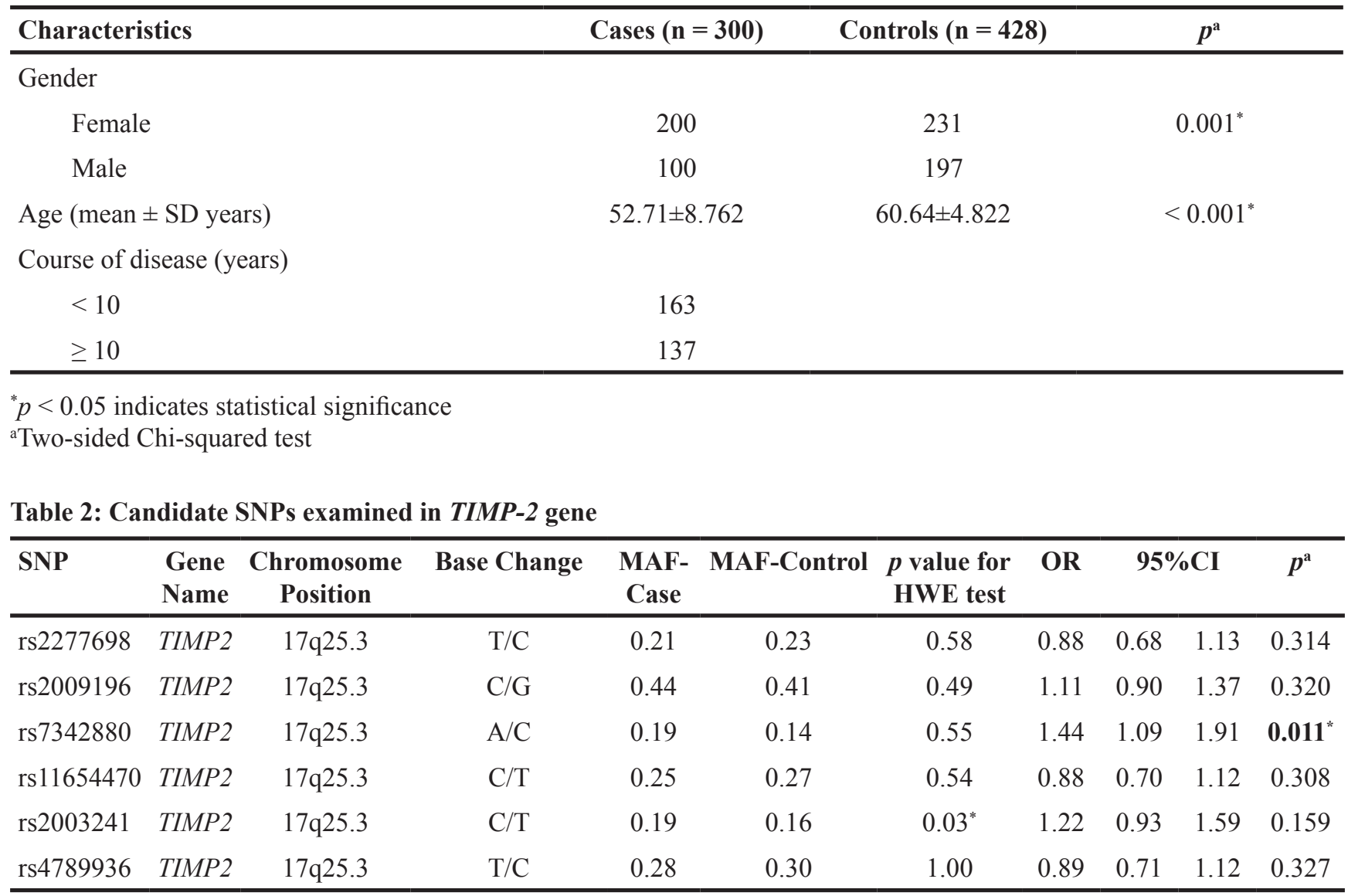

$\mathrm{SNP}=$ single nucleotide polymorphism; MAF $=$ minor allele frequency; $\mathrm{OR}=$ odds ratio; $95 \% \mathrm{CI}=95 \%$ confidence interval;

$\mathrm{HWE}=$ Hardy-Weinberg equilibrium;

${ }^{*} p<0.05$ indicates statistical significance;

$p$ value were calculated using two-sided Chi-squared test.

Table 3: Frequency distributions of SNPs in TIMP-2 and their associations with the risk of developing knee OA under multiple models of inheritance

\begin{tabular}{|c|c|c|c|c|c|c|c|c|}
\hline \multirow[t]{2}{*}{ SNP } & \multirow[t]{2}{*}{ Model } & \multirow[t]{2}{*}{ Genotype } & \multirow{2}{*}{$\begin{array}{l}\text { Control } \\
\text { N }(\%)\end{array}$} & \multirow{2}{*}{$\begin{array}{c}\text { Case } \\
\text { N }(\%)\end{array}$} & \multicolumn{2}{|c|}{ Adjustment analysis } & \multicolumn{2}{|c|}{ Crude analysis } \\
\hline & & & & & $\begin{array}{l}\text { OR }(95 \% \\
\text { CI })\end{array}$ & $p^{\mathbf{a}}$ & $\begin{array}{l}\text { OR }(95 \% \\
\text { CI) }\end{array}$ & $p^{b}$ \\
\hline \multirow[t]{5}{*}{ rs2277698 } & Codominant & $\mathrm{C} / \mathrm{C}$ & $251(58.9 \%)$ & $187(62.3 \%)$ & 1 & 0.400 & 1 & 0.590 \\
\hline & & $\mathrm{C} / \mathrm{T}$ & $155(36.4 \%)$ & $102(34 \%)$ & $\begin{array}{c}0.82(0.57- \\
1.17)\end{array}$ & & $\begin{array}{c}0.88(0.65- \\
1.21)\end{array}$ & \\
\hline & & $\mathrm{T} / \mathrm{T}$ & $20(4.7 \%)$ & $11(3.7 \%)$ & $\begin{array}{c}0.66(0.28- \\
1.59)\end{array}$ & & $\begin{array}{c}0.74(0.35- \\
1.58)\end{array}$ & \\
\hline & Dominant & $\mathrm{C} / \mathrm{C}$ & $251(58.9 \%)$ & $187(62.3 \%)$ & 1 & 0.210 & 1 & 0.350 \\
\hline & & $\mathrm{C} / \mathrm{T}-\mathrm{T} / \mathrm{T}$ & $175(41.1 \%)$ & $113(37.7 \%)$ & $\begin{array}{c}0.80(0.56- \\
1.13)\end{array}$ & & $\begin{array}{c}0.87(0.64- \\
1.17)\end{array}$ & \\
\hline
\end{tabular}




\begin{tabular}{|c|c|c|c|c|c|c|c|c|}
\hline \multirow[t]{2}{*}{ SNP } & \multirow[t]{2}{*}{ Model } & \multirow[t]{2}{*}{ Genotype } & \multirow{2}{*}{$\begin{array}{c}\text { Control } \\
\text { N (\%) }\end{array}$} & \multirow{2}{*}{$\begin{array}{l}\text { Case } \\
\text { N (\%) }\end{array}$} & \multicolumn{2}{|c|}{ Adjustment analysis } & \multicolumn{2}{|c|}{ Crude analysis } \\
\hline & & & & & $\begin{array}{c}\text { OR }(95 \% \\
\text { CI) }\end{array}$ & $p^{\mathrm{a}}$ & $\begin{array}{c}\text { OR }(95 \% \\
\text { CI) }\end{array}$ & $p^{\mathrm{b}}$ \\
\hline & Over- & $\mathrm{C} / \mathrm{C}-\mathrm{T} / \mathrm{T}$ & $271(63.6 \%)$ & $198(66 \%)$ & 1 & 0.330 & 1 & 0.510 \\
\hline & & $\mathrm{C} / \mathrm{T}$ & $155(36.4 \%)$ & $102(34 \%)$ & $\begin{array}{c}0.84(0.59- \\
1.19)\end{array}$ & & $\begin{array}{c}0.90(0.66- \\
1.23)\end{array}$ & \\
\hline & Log-additive & --- & --- & --- & $\begin{array}{c}0.82(0.61- \\
1.10)\end{array}$ & 0.180 & $\begin{array}{c}0.87(0.67- \\
1.13)\end{array}$ & 0.310 \\
\hline \multirow[t]{10}{*}{ rs2009196 } & Codominant & $\mathrm{G} / \mathrm{G}$ & $151(35.4 \%)$ & $94(31.3 \%)$ & 1 & 0.880 & 1 & 0.530 \\
\hline & & $\mathrm{C} / \mathrm{G}$ & $200(46.8 \%)$ & $149(49.7 \%)$ & $\begin{array}{c}1.07(0.73- \\
1.57)\end{array}$ & & $\begin{array}{c}1.20(0.86- \\
1.67)\end{array}$ & \\
\hline & & $\mathrm{C} / \mathrm{C}$ & $76(17.8 \%)$ & $57(19 \%)$ & $\begin{array}{c}0.96(0.58- \\
1.59)\end{array}$ & & $\begin{array}{c}1.20(0.78- \\
1.85)\end{array}$ & \\
\hline & Dominant & $\mathrm{G} / \mathrm{G}$ & $151(35.4 \%)$ & $94(31.3 \%)$ & 1 & 0.830 & 1 & 0.260 \\
\hline & & $\mathrm{C} / \mathrm{G}-\mathrm{C} / \mathrm{C}$ & $276(64.6 \%)$ & $206(68.7 \%)$ & $\begin{array}{c}1.04(0.72- \\
1.50)\end{array}$ & & $\begin{array}{c}1.20(0.88- \\
1.64)\end{array}$ & \\
\hline & Recessive & $\mathrm{G} / \mathrm{G}-\mathrm{C} / \mathrm{G}$ & $351(82.2 \%)$ & $243(81 \%)$ & 1 & 0.720 & 1 & 0.680 \\
\hline & & $\mathrm{C} / \mathrm{C}$ & $76(17.8 \%)$ & $57(19 \%)$ & $\begin{array}{c}0.92(0.59- \\
1.43)\end{array}$ & & $\begin{array}{c}1.08(0.74- \\
1.58)\end{array}$ & \\
\hline & Over- & $\mathrm{G} / \mathrm{G}-\mathrm{C} / \mathrm{C}$ & $227(53.2 \%)$ & $151(50.3 \%)$ & 1 & 0.640 & 1 & 0.450 \\
\hline & & $\mathrm{C} / \mathrm{G}$ & $200(46.8 \%)$ & $149(49.7 \%)$ & $\begin{array}{c}1.08(0.77- \\
1.52)\end{array}$ & & $\begin{array}{c}1.12(0.83- \\
1.51)\end{array}$ & \\
\hline & Log-additive & --- & --- & --- & $\begin{array}{c}0.99(0.78- \\
1.27)\end{array}$ & 0.950 & $\begin{array}{c}1.11(0.90- \\
1.37)\end{array}$ & 0.320 \\
\hline \multirow[t]{10}{*}{ rs 7342880} & Codominant & $\mathrm{C} / \mathrm{C}$ & $318(74.3 \%)$ & $199(66.3 \%)$ & 1 & 0.180 & 1 & $0.046^{*}$ \\
\hline & & $\mathrm{C} / \mathrm{A}$ & $100(23.4 \%)$ & $88(29.3 \%)$ & $\begin{array}{c}1.29(0.88- \\
1.90)\end{array}$ & & $\begin{array}{c}1.41(1.00- \\
1.97)\end{array}$ & \\
\hline & & $\mathrm{A} / \mathrm{A}$ & $10(2.3 \%)$ & $13(4.3 \%)$ & $\begin{array}{c}2.02(0.77- \\
5.32)\end{array}$ & & $\begin{array}{c}2.08(0.89- \\
4.83)\end{array}$ & \\
\hline & Dominant & $\mathrm{C} / \mathrm{C}$ & $318(74.3 \%)$ & $199(66.3 \%)$ & 1 & 0.110 & 1 & $0.020^{*}$ \\
\hline & & $\mathrm{C} / \mathrm{A}-\mathrm{A} / \mathrm{A}$ & $110(25.7 \%)$ & $101(33.7 \%)$ & $\begin{array}{c}1.36(0.94- \\
1.96)\end{array}$ & & $\begin{array}{c}1.47(1.06- \\
2.03)\end{array}$ & \\
\hline & Recessive & $\mathrm{C} / \mathrm{C}-\mathrm{C} / \mathrm{A}$ & 418 (97.7\%) & 287 (95.7\%) & 1 & 0.190 & 1 & 0.130 \\
\hline & & $\mathrm{A} / \mathrm{A}$ & $10(2.3 \%)$ & $13(4.3 \%)$ & $\begin{array}{c}1.88(0.72- \\
4.92)\end{array}$ & & $\begin{array}{c}1.89(0.82- \\
4.38)\end{array}$ & \\
\hline & $\begin{array}{c}\text { Over- } \\
\text { dominant }\end{array}$ & $\mathrm{C} / \mathrm{C}-\mathrm{A} / \mathrm{A}$ & $328(76.6 \%)$ & $212(70.7 \%)$ & 1 & 0.250 & 1 & 0.071 \\
\hline & & $\mathrm{C} / \mathrm{A}$ & $100(23.4 \%)$ & $88(29.3 \%)$ & $\begin{array}{c}1.25(0.85- \\
1.83)\end{array}$ & & $\begin{array}{c}1.36(0.97- \\
1.90)\end{array}$ & \\
\hline & Log-additive & --- & --- & --- & $\begin{array}{c}1.34(0.98- \\
1.84)\end{array}$ & 0.069 & $\begin{array}{c}1.42(1.08- \\
1.87)\end{array}$ & $0.013^{*}$ \\
\hline rs11654470 & Codominant & $\mathrm{T} / \mathrm{T}$ & $224(52.3 \%)$ & $168(56 \%)$ & 1 & 0.250 & 1 & 0.580 \\
\hline
\end{tabular}




\begin{tabular}{|c|c|c|c|c|c|c|c|c|}
\hline \multirow[t]{2}{*}{ SNP } & \multirow[t]{2}{*}{ Model } & \multirow[t]{2}{*}{ Genotype } & \multirow{2}{*}{$\begin{array}{c}\text { Control } \\
\text { N (\%) }\end{array}$} & \multirow{2}{*}{$\begin{array}{c}\text { Case } \\
\text { N (\%) }\end{array}$} & \multicolumn{2}{|c|}{ Adjustment analysis } & \multicolumn{2}{|c|}{ Crude analysis } \\
\hline & & & & & $\begin{array}{c}\text { OR }(95 \% \\
\text { CI) }\end{array}$ & $p^{\mathrm{a}}$ & $\begin{array}{c}\text { OR }(95 \% \\
\text { CI) }\end{array}$ & $p^{b}$ \\
\hline & & $\mathrm{T} / \mathrm{C}$ & $175(40.9 \%)$ & $115(38.3 \%)$ & $\begin{array}{c}0.78(0.55- \\
1.12)\end{array}$ & & $\begin{array}{c}0.88(0.64- \\
1.19)\end{array}$ & \\
\hline & & $\mathrm{C} / \mathrm{C}$ & $29(6.8 \%)$ & $17(5.7 \%)$ & $\begin{array}{c}0.63(0.31- \\
1.31)\end{array}$ & & $\begin{array}{c}0.78(0.42- \\
1.47)\end{array}$ & \\
\hline & Dominant & $\mathrm{T} / \mathrm{T}$ & $224(52.3 \%)$ & $168(56 \%)$ & 1 & 0.120 & 1 & 0.330 \\
\hline & & $\mathrm{T} / \mathrm{C}-\mathrm{C} / \mathrm{C}$ & $204(47.7 \%)$ & $132(44 \%)$ & $\begin{array}{c}0.76(0.54- \\
1.07)\end{array}$ & & $\begin{array}{c}0.86(0.64- \\
1.16)\end{array}$ & \\
\hline & Recessive & $\mathrm{T} / \mathrm{T}-\mathrm{T} / \mathrm{C}$ & $399(93.2 \%)$ & $283(94.3 \%)$ & 1 & 0.330 & 1 & 0.540 \\
\hline & & $\mathrm{C} / \mathrm{C}$ & $29(6.8 \%)$ & $17(5.7 \%)$ & $\begin{array}{c}0.71(0.35- \\
1.43)\end{array}$ & & $\begin{array}{c}0.83(0.45- \\
1.53)\end{array}$ & \\
\hline & $\begin{array}{c}\text { Over- } \\
\text { dominant }\end{array}$ & $\mathrm{T} / \mathrm{T}-\mathrm{C} / \mathrm{C}$ & $253(59.1 \%)$ & $185(61.7 \%)$ & 1 & 0.260 & 1 & 0.490 \\
\hline & & $\mathrm{T} / \mathrm{C}$ & $175(40.9 \%)$ & $115(38.3 \%)$ & $\begin{array}{c}0.82(0.58- \\
1.16)\end{array}$ & & $\begin{array}{c}0.90(0.66- \\
1.22)\end{array}$ & \\
\hline & Log-additive & --- & --- & --- & $\begin{array}{c}0.79(0.60- \\
1.04)\end{array}$ & 0.096 & $\begin{array}{c}0.88(0.69- \\
1.12)\end{array}$ & 0.300 \\
\hline \multirow[t]{10}{*}{ rs 2003241} & Codominant & $\mathrm{T} / \mathrm{T}$ & $305(71.3 \%)$ & $197(65.7 \%)$ & 1 & 0.520 & 1 & 0.580 \\
\hline & & $\mathrm{T} / \mathrm{C}$ & $105(24.5 \%)$ & $90(30 \%)$ & $\begin{array}{c}1.24(0.84- \\
1.81)\end{array}$ & & $\begin{array}{c}0.88(0.64- \\
1.19)\end{array}$ & \\
\hline & & $\mathrm{C} / \mathrm{C}$ & $18(4.2 \%)$ & $13(4.3 \%)$ & $\begin{array}{c}0.91(0.40- \\
2.08)\end{array}$ & & $\begin{array}{c}0.78(0.42- \\
1.47)\end{array}$ & \\
\hline & Dominant & $\mathrm{T} / \mathrm{T}$ & $305(71.3 \%)$ & $197(65.7 \%)$ & 1 & 0.360 & 1 & 0.110 \\
\hline & & $\mathrm{T} / \mathrm{C}-\mathrm{C} / \mathrm{C}$ & $123(28.7 \%)$ & $103(34.3 \%)$ & $\begin{array}{c}1.18(0.82- \\
1.70)\end{array}$ & & $\begin{array}{c}1.30(0.94- \\
1.78)\end{array}$ & \\
\hline & Recessive & $\mathrm{T} / \mathrm{T}-\mathrm{T} / \mathrm{C}$ & $410(95.8 \%)$ & $287(95.7 \%)$ & 1 & 0.710 & 1 & 0.930 \\
\hline & & $\mathrm{C} / \mathrm{C}$ & $18(4.2 \%)$ & $13(4.3 \%)$ & $\begin{array}{c}0.86(0.38- \\
1.94)\end{array}$ & & $\begin{array}{c}1.03(0.50- \\
2.14)\end{array}$ & \\
\hline & $\begin{array}{c}\text { Over- } \\
\text { dominant }\end{array}$ & $\mathrm{T} / \mathrm{T}-\mathrm{C} / \mathrm{C}$ & $323(75.5 \%)$ & $210(70 \%)$ & 1 & 0.260 & 1 & 0.100 \\
\hline & & $\mathrm{T} / \mathrm{C}$ & $105(24.5 \%)$ & $90(30 \%)$ & $\begin{array}{c}1.24(0.85- \\
1.82)\end{array}$ & & $\begin{array}{c}1.32(0.95- \\
1.84)\end{array}$ & \\
\hline & Log-additive & --- & --- & --- & $\begin{array}{c}1.10(0.81- \\
1.48)\end{array}$ & 0.540 & $\begin{array}{c}1.20(0.92- \\
1.56)\end{array}$ & 0.180 \\
\hline \multirow[t]{5}{*}{ rs4789936 } & Codominant & $\mathrm{C} / \mathrm{C}$ & $208(48.6 \%)$ & $160(54 \%)$ & 1 & 0.110 & 1 & 0.250 \\
\hline & & $\mathrm{C} / \mathrm{T}$ & $181(42.3 \%)$ & $107(36.1 \%)$ & $\begin{array}{c}0.68(0.47- \\
0.98)\end{array}$ & & $\begin{array}{c}0.77(0.56- \\
1.05)\end{array}$ & \\
\hline & & $\mathrm{T} / \mathrm{T}$ & $39(9.1 \%)$ & $29(9.8 \%)$ & $\begin{array}{c}0.92(0.50- \\
1.69)\end{array}$ & & $\begin{array}{c}0.97(0.57- \\
1.63)\end{array}$ & \\
\hline & Dominant & $\mathrm{C} / \mathrm{C}$ & $208(48.6 \%)$ & $160(54 \%)$ & 1 & 0.058 & 1 & 0.150 \\
\hline & & $\mathrm{C} / \mathrm{T}-\mathrm{T} / \mathrm{T}$ & $220(51.4 \%)$ & $136(46 \%)$ & $\begin{array}{c}0.72(0.51- \\
1.01)\end{array}$ & & $\begin{array}{c}0.80(0.60- \\
1.08)\end{array}$ & \\
\hline
\end{tabular}




\begin{tabular}{|c|c|c|c|c|c|c|c|c|}
\hline \multirow[t]{2}{*}{ SNP } & \multirow[t]{2}{*}{ Model } & \multirow[t]{2}{*}{ Genotype } & \multirow{2}{*}{$\begin{array}{c}\text { Control } \\
\text { N }(\%)\end{array}$} & \multirow{2}{*}{$\begin{array}{c}\text { Case } \\
\text { N }(\%)\end{array}$} & \multicolumn{2}{|c|}{ Adjustment analysis } & \multicolumn{2}{|c|}{ Crude analysis } \\
\hline & & & & & $\begin{array}{c}\text { OR }(95 \% \\
\text { CI) }\end{array}$ & $p^{\mathrm{a}}$ & $\begin{array}{c}\text { OR }(95 \% \\
\text { CI })\end{array}$ & $p^{\mathrm{b}}$ \\
\hline & Recessive & $\mathrm{C} / \mathrm{C}-\mathrm{C} / \mathrm{T}$ & $389(90.9 \%)$ & $267(90.2 \%)$ & 1 & 0.790 & 1 & 0.760 \\
\hline & & $\mathrm{T} / \mathrm{T}$ & $39(9.1 \%)$ & $29(9.8 \%)$ & $\begin{array}{c}1.09(0.60- \\
1.96)\end{array}$ & & $\begin{array}{c}1.08(0.65- \\
1.80)\end{array}$ & \\
\hline & $\begin{array}{c}\text { Over- } \\
\text { dominant }\end{array}$ & $\mathrm{C} / \mathrm{C}-\mathrm{T} / \mathrm{T}$ & $247(57.7 \%)$ & $189(63.9 \%)$ & 1 & $0.036^{*}$ & 1 & 0.096 \\
\hline & & $\mathrm{C} / \mathrm{T}$ & $181(42.3 \%)$ & $107(36.1 \%)$ & $\begin{array}{c}0.69(0.49- \\
0.98)\end{array}$ & & $\begin{array}{c}0.77(0.57- \\
1.05)\end{array}$ & \\
\hline & Log-additive & --- & --- & --- & $\begin{array}{c}0.84(0.64- \\
1.09)\end{array}$ & 0.180 & $\begin{array}{c}0.89(0.71- \\
1.12)\end{array}$ & 0.340 \\
\hline
\end{tabular}

${ }^{*} p<0.05$ indicates statistical significance; $\mathrm{OR}=$ odds ratio; $95 \% \mathrm{CI}=95 \%$ confidence interval;

${ }^{a} p$ values were calculated by unconditional logistic regression adjusted for age and gender

${ }^{\mathrm{b}} p$ values were calculated without adjustment for age and gender

Table 4: Frequency distributions of rs7342880 SNP and its association with the risk of developing knee OA

\begin{tabular}{lccccc}
\hline Variable & \multicolumn{5}{c}{ rs7342880 } \\
\hline & CC & CA+AA & $\boldsymbol{p}$ & OR & $\mathbf{9 5 \% / C I ~}$ \\
\hline $\begin{array}{c}\text { Age (year) } \\
>60\end{array}$ & 101 & 51 & -- & 1.00 & (reference) \\
$\quad \leq 60$ & 98 & 50 & 0.966 & 0.99 & $0.613-1.598$ \\
Course of disease (year) & & & & \\
$\quad<10$ & 112 & 51 & -- & 1.00 & (reference) \\
$\geq 10$ & 87 & 50 & 0.342 & 1.262 & $0.781-2.040$ \\
Gender & & & & \\
$\quad$ Male & 74 & 26 & --- & 1.00 & (reference) \\
Female & 125 & 75 & $\mathbf{0 . 0 4 8}$ & 1.708 & $1.004-2.903$ \\
\hline
\end{tabular}

$\mathrm{OR}=$ odds ratio $; 95 \% \mathrm{CI}=95 \%$ confidence interval;

${ }^{*} p<0.05$ indicates statistical significance;

$p$ value were calculated using two-sided Chi-squared test

$=1.00-2.39)$. We detected no significant interactions between these and other SNPs examined (data not shown).

\section{DISCUSSION}

Cartilage degradation in knee OA involves MMP activity as collagenases (MMP-1 and 13), gelatinases (MMP-2 and MMP-9) and stromelysin (MMP-3) [20]. Of the three major MMPs that degrade native collagen (MMP-1, MMP-8, and MMP-13), recent work has shown MMP-13 was the most important in knee OA due to its preferential degradation of type II collagen [21]. Furthermore, increased expression of MMP-13 is observed in knee OA [22]. Nevertheless, all active MMPs are inhibited by TIMPs. TIMP-2 is a non-glycosylated protein, which blocks the hydrolase activity of all MMPs [23], including MMP-13. In addition, in vitro experiments demonstrated an association between TIMP-2 and osteopontin, involving pathological changes of knee OA [18].

TIMP-2 gene polymorphisms have been studied in several diseases such as hypertension [24], Achilles tendon pathologies [25], cancer [26], and varicose vein [27]. In the current study, we studied six TIMP-2 gene polymorphisms in Chinese patients with knee OA and healthy controls. We found that rs7342880, an intronic SNP within the TIMP-2 gene, was significantly associated with knee OA risk according to allele association analysis. A similar 
Table 5: TIMP-2 haplotype frequencies and the association with knee $O A$ risk in case and control subjects

\begin{tabular}{|c|c|c|c|c|c|c|c|c|c|c|}
\hline \multirow[t]{2}{*}{ rs2277698 } & \multirow[t]{2}{*}{ rs2009196 } & \multirow[t]{2}{*}{ rs7342880 } & \multirow[t]{2}{*}{ rs11654470 } & \multicolumn{2}{|c|}{ Frequence (\%) } & \multirow[t]{2}{*}{$\chi^{2}$} & \multicolumn{2}{|c|}{ Crude Analysis } & \multicolumn{2}{|c|}{ Adjusted Analysis } \\
\hline & & & & Case & Control & & OR $(95 \%$ CI $)$ & $p^{\mathrm{a}}$ & OR $(95 \%$ CI $)$ & $p^{\mathrm{b}}$ \\
\hline $\mathrm{C}$ & G & $\mathrm{C}$ & $\mathrm{T}$ & 0.564 & 0.584 & 0.632 & 1 & --- & 1 & --- \\
\hline $\mathrm{T}$ & $\mathrm{C}$ & $\mathrm{C}$ & $\mathrm{C}$ & 0.207 & 0.229 & 0.916 & $0.93(0.71-1.22)$ & 0.620 & $0.85(0.62-1.16)$ & 0.310 \\
\hline $\mathrm{C}$ & $\mathrm{C}$ & A & $\mathrm{T}$ & 0.187 & 0.14 & 5.949 & $1.39(1.04-1.86)$ & $0.025^{*}$ & $1.27(0.91-1.77)$ & 0.160 \\
\hline $\mathrm{C}$ & $\mathrm{C}$ & $\mathrm{C}$ & $\mathrm{C}$ & 0.04 & 0.041 & 0.015 & $0.99(0.57-1.71)$ & 0.960 & $0.74(0.39-1.39)$ & 0.350 \\
\hline
\end{tabular}

$\mathrm{OR}=$ odds ratio; $95 \% \mathrm{CI}=95 \%$ confidence interval;

" $p<0.05$ indicates statistical significance;

${ }^{a} p$ value from were calculated from two-sided Chi-squared test without adjusted by age and gender;

${ }^{\mathrm{b}} p$ values were calculated by unconditional logistic regression adjusted for age and sex.

Table 6: Significant associations between SNPs and knee OA after stratification by gender

\begin{tabular}{|c|c|c|c|c|c|c|c|c|c|c|c|c|}
\hline \multirow[t]{3}{*}{ Genotype } & \multicolumn{6}{|c|}{ Female } & \multicolumn{6}{|c|}{ Male } \\
\hline & \multicolumn{2}{|c|}{ case } & \multicolumn{2}{|c|}{ control } & \multirow[t]{2}{*}{ OR $(95 \%$ CI $)$} & \multirow[t]{2}{*}{$p^{\mathrm{a}}$} & \multicolumn{2}{|c|}{ case } & \multicolumn{2}{|c|}{ control } & \multirow[t]{2}{*}{ OR (95 \% CI) } & \multirow[t]{2}{*}{$p^{\mathrm{a}}$} \\
\hline & $\mathbf{N}$ & $\%$ & $\mathbf{N}$ & $\%$ & & & $\mathbf{N}$ & $\%$ & $\mathbf{N}$ & $\%$ & & \\
\hline $\mathrm{C} / \mathrm{C}$ & 125 & 62.5 & 171 & 74 & 1 [Ref] & & 74 & 74 & 147 & 74.6 & 1 [Ref] & \\
\hline $\mathrm{C} / \mathrm{A}$ & 67 & 33.5 & 54 & 23.4 & $1.52(0.97-2.39)$ & 0.140 & 21 & 21 & 46 & 23.4 & $0.96(0.44-2.06)$ & 0.580 \\
\hline $\mathrm{A} / \mathrm{A}$ & 8 & 4 & 6 & 2.6 & $1.80(0.58-5.52)$ & & 5 & 5 & 4 & 2 & $3.02(0.35-25.91)$ & \\
\hline $\mathrm{C} / \mathrm{A}-\mathrm{A} / \mathrm{A}$ & 75 & 37.5 & 60 & 26 & $1.55(1.00-2.39)$ & $0.048^{*}$ & 26 & 26 & 50 & 25.4 & $1.07(0.51-2.22)$ & 0.860 \\
\hline
\end{tabular}

$\mathrm{OR}=$ odds ratio; $95 \% \mathrm{CI}=95 \%$ confidence interval; $[\mathrm{Ref}]=$ reference category;

${ }^{*} p<0.05$ indicates statistical significance;

${ }^{a} p$ value from were calculated from two-sided Chi-squared test adjusted by age and gender

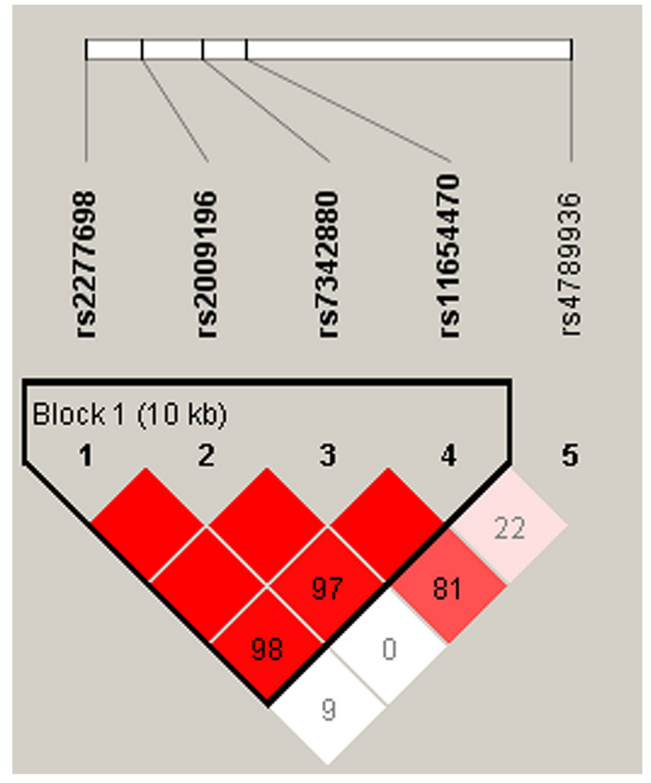

Figure 1: linkage disequilibrium of polymorphic sites in the TIMP-2 gene. 
distribution of other polymorphisms was observed in both studied groups. "A" allele of rs7342880 was ascertained to be associated with increased risk for the development of knee OA. Similar to our results, rs7342880 has also been associated with increased risk of knee OA in a previous study involving a Spanish population [28]. This study is the first genotype/allele-based study that describes the association between SNPs within the TIMP-2 locus and knee OA risk in a Chinese population.

We found another polymorphism in TIMP-2 rs4789936 associated with decreased risk of knee OA by the over-dominant model. A previous study found that rs4789936 SNP in TIMP-2 gene may be involved in breast cancer susceptibility and survival in a Shanghaibased population [29]. Our study is the first report on the association between the rs4789936 SNP in TIMP-2 and knee OA risk in any Chinese population. Further studies to determine the mechanism of rs4789936 should be considered.

Haplotype analysis suggested that knee OA risk was substantially elevated among individuals with specific haplotypes. Before adjustment, "CCAT" was a risk haplotype $(\mathrm{OR}=1.39)$. After adjustment for age and gender, we found no statistically significant association between these TIMP-2 SNPs and knee OA risk. The mechanism responsible for this finding is unclear. This trend did not reach statistical significance, perhaps because of the small number of patients included.

By stratification analysis in gender-specific populations, we found that the "CA+AA" genotype of rs7342880 was associated with increased risk of knee OA in females compared to the "C/C" genotype. Similarly, epidemiological studies have shown that females have an increased risk of OA occurrence in the hand, knee, and in multiple joints [30], which may help explain the sex differences in the prevalence of OA. In addition, gene polymorphisms were analyzed to establish their associations with clinicopathological features, including gender, age, and duration of knee OA (years). rs7342880 was associated with increased risk of knee OA (without adjustment, "CA+AA" $\mathrm{OR}=1.708 ; 95 \% \mathrm{CI}=1.004$ $2.903 ; p=0.048$ ), consistent with stratification analysis. The accelerated rise in knee OA incidence in women following menopause suggests a possible role for sex hormones, particularly estrogen deficiency. However, there is no consistent evidence linking circulating sex hormone levels $[31,32]$ with OA prevalence. Therefore, the results of the association between gene polymorphisms and the risk of knee OA in females were stable, because we excluded the effects of sex hormones.

Some inherent limitations of this case-control study must be noted. First, the sample size is relatively small relative to knee OA association studies published to date [33-35]. Additionally, body mass index (BMI) is a known confounding factor for association analysis and affects the onset or progress of knee OA [36, 37]. In this study, knee OA patients and controls were not adjusted for BMI. In future studies, to uncover the role of BMI between genetic variants and the risk of knee OA, BMI should be recorded in the same OA subjects or even the more samples. In addition, the results of our population comparison by the $\chi^{2}$ test indicated that there are some differences in gender and age. Multiple independent studies with large sample sizes and gathering detailed clinical information are needed to validate our findings.

In conclusion, our comprehensive analysis, for the first time, suggests that SNPs (rs7342880 and rs4789936) in TIMP-2 genotype and allele are associated with knee OA risk in Chinese Han population. Identifying the underlying genetic factors could enhance our understanding of the pathogenesis of this complex disorder, which could eventually contribute to improved prevention and treatment of this common disease. Further studies are needed in large cohorts of knee OA patients including the polymorphisms of other TIMPS (i.e., TIMP1, TIMP-4) genes.

\section{MATERIALS AND METHODS}

\section{Subject recruitment and sample collection}

We recruited 300 Han Chinese patients diagnosed with knee OA for this case-control study from February 2013 to July 2015. All patients were diagnosed by the Second Department of Trauma \& Hand and Foot Surgery, Second Affiliated Hospital, Inner Mongolia Medical University, China. The diagnosis of knee OA was based on a detailed history, physical exam, and/or radiographic studies. Diagnostic criteria developed by the American College of Rheumatology (ACR) include the presence of knee joint pain, osteophytes or bone spurs on X-ray, and one or more associated symptoms in the knee joint. Other associated symptoms include knee stiffness, crepitus on physical exam, point tenderness, enlargement or deformity of the knee joint, and a narrowing knee joint space noted on radiography [38]. In our study, we used broad inclusion criteria for primary knee OA cases with any symptoms and signs of $\mathrm{OA}$, and radiographic signs of knee OA according to the Kellgren-Lawrence grading. Additionally, participants who had undergone total knee replacement with a radiological score $>2$ on the Kellgren and Lawrence scale were included. Exclusion criteria were other etiologies causing knee diseases such as inflammatory arthritis (rheumatoid, polyarthritis, or autoimmune disease), post-traumatic or post-septic arthritis, skeletal dysplasia, or developmental dysplasia.

The control group included 428 individuals who had never exhibited any signs or symptoms of knee OA, other arthritis, or joint diseases (pain, swelling, tenderness, or restriction of movement) at any site, based 
Table 7: PCR primers for SNPs used in this study

\begin{tabular}{llcc}
\hline SNP & 1st_PCRP & 2st_PCRP & UEP_SEQ \\
\hline rs2277698 & ACGTTGGATGAGAGT & ACGTTGGATGTCATAC & TTCTTTCCTCC \\
& TTATCTACACGGCCC & ACACCTGCAATGAG & AACGTCCAG \\
rs2009196 & ACGTTGGATGACCTT & ACGTTGGATGGAGTTA & AACGTACCCG \\
& TCCAGATGTAAGACC & TCCACCTTAAAGGG & GCATATTTAG \\
rs7342880 & ACGTTGGATGTGCGG & ACGTTGGATGATTCG & ccTTCGCCCTGCTT \\
& TGCCCGGGAACTAAT & CCCTGCTTGTCTATG & GTCTATGCGATGC \\
rs2003241 & ACGTTGGATGTTCTG & ACGTTGGATGATAAGC & CCCTGCCAGT \\
& CAGGCTCCAGCTTTC & AGGACAGGACAGAG & GCTGGTCCT \\
rs4789936 & ACGTTGGATGGCAAA & ACGTTGGATGAAATGA & GACTCTACAA \\
& ACTAGGCACAAAGGG & AAGGGCGTGGCCAG & AAATAGGTGGTG \\
& TCACTACCTACAAAG & ACGTTGGATGAACAC & aTCTCCAGGGCTG \\
& & ATTACAGGGAGGCAG & TCTTGAAATCA \\
\hline
\end{tabular}

on their medical history and a thorough examination conducted by an experienced physiatrist. In addition, patients who had undergone treatment for injuries and fractures, with no symptoms or clinical signs of OA were also included.

All individuals were of Chinese origin. This study was approved by the ethics committee of Inner Mongolia Medical University and written informed consent was obtained from all participants.

\section{SNP selection and genotyping}

Genetic variants were randomly selected from the TIMP-2 gene annotated by the HapMap database. Six candidate SNPs (rs2277698, rs2009196, rs7342880, rs 11654470, rs2003241, rs4789936) in the TIMP-2 gene with minor allele frequencies (MAF) $>5 \%$ in Asians were finally selected for genotyping. Five milliliters of venous blood were collected in ethylenediamine-tetra-acetic acid (EDTA) containing tubes and stored at $-20^{\circ} \mathrm{C}$. DNA was extracted from whole blood using a GoldMag-Mini Whole Blood Genomic DNA Purification Kit (GoldMag Ltd., Xi'an, China) according to the manufacturer's protocol, and the concentration of DNA was measured by NanoDrop 2000C (Thermo Scientific, Waltham, MA). The Sequenom MassARRAY Assay Design 3.0 Software (San Diego, CA) was used to design Multiplexed SNP MassEXTEND assays. PCR primers used for each SNP are listed in Table 7. SNPs were genotyped using Sequenom MassARRAY RS1000 (San Diego, CA) according to the standard protocol recommended by the manufacturer [39]. Polymorphism proliferation was carried out by standard polymerase chain reaction and restriction fragment length polymorphism (PCR-RLFP) as previously described
[40]. We applied Sequenom Typer 4.0 Software (San Diego, CA) to perform data management and analyses as described [41].

\section{Statistical analysis}

Microsoft Excel and SPSS 19.0 statistical packages (SPSS, Chicago, IL) were used to perform statistical analyses. A probability value $p$ less than 0.05 was considered statistically significant. Allele frequency of each SNP in the control subjects was analyzed using Chi-squared $\left(\chi^{2}\right)$ test to evaluate departure from HardyWeinberg equilibrium (HWE). The $\chi^{2}$ test was also used to compare the genotype and allele distributions for SNPs between the control and the knee OA groups [42]. Five genetic models (co-dominant, dominant, recessive, over-dominant, and additive) were applied by PLINK software (http://pngu.mgh.harvard.edu/purcell/plink/). Unconditional logistic regression, adjusted for age and gender, was used to estimate relative risk of knee OA for each of the tested genotype in the form of odds ratio (OR) and $95 \%$ confidence intervals (CIs)[43]. We used Haploview software package (version 4.2) to perform the analysis for linkage disequilibrium (LD), constructed haplotypes and genetic association at significant polymorphism loci.

\section{ACKNOWLEDGMENTS}

The authors wish to thank participants in the study who made this work possible. We would also like to thank the clinicians and other hospital staff who contributed to the blood sample and data collection for this study. 


\section{CONFLICTS OF INTEREST}

All authors declare no conflict of interest.

\section{FINANCIAL SUPPORT}

This work was supported by the National Natural Science Foundation of China (nos. 81160228, 81260284 and 81660378).

\section{REFERENCES}

1. Wang T, Liang Y, Li H, Li H, He Q, Xue Y, Shen C, Zhang C, Xiang J, Ding J, Qiao L and Zheng Q. Single Nucleotide Polymorphisms and Osteoarthritis: An Overview and a Meta-Analysis. Medicine. 2016; 95:e2811.

2. Uitterlinden $\mathrm{AG}$, Ralston $\mathrm{SH}$, Brandi ML, Carey $\mathrm{AH}$, Grinberg D, Langdahl BL, Lips P, Lorenc R, ObermayerPietsch B, Reeve J, Reid DM, Amedei A, Bassiti A, Bustamante M, Husted LB, Diez-Perez A, et al. The association between common vitamin $\mathrm{D}$ receptor gene variations and osteoporosis: a participant-level metaanalysis. Annals of internal medicine. 2006; 145:255-264.

3. Simon LS. Osteoarthritis: a review. Clinical cornerstone. 1999; 2:26-37.

4. Lepetsos P, Pampanos A, Kanavakis E, Tzetis M, Korres D, Papavassiliou AG and Efstathopoulos N. Association of MMP-1 -1607 1G/2G (rs1799750) polymorphism with primary knee osteoarthritis in the Greek population. Journal of orthopaedic research. 2014; 32:1155-1160.

5. Somerville RP, Oblander SA and Apte SS. Matrix metalloproteinases: old dogs with new tricks. Genome Biology. 2003; 4:1-11.

6. Birkedal-Hansen H, Moore W, Bodden M, Windsor L, Birkedal-Hansen B, DeCarlo A and Engler J. Matrix metalloproteinases: a review. Critical Reviews in Oral Biology \& Medicine. 1993; 4:197-250.

7. Barlas IO, Sezgin M, Erdal ME, Sahin G, Ankarali HC, Altintas ZM and Turkmen E. Association of $(-1,607)$ $1 \mathrm{G} / 2 \mathrm{G}$ polymorphism of matrix metalloproteinase-1 gene with knee osteoarthritis in the Turkish population (knee osteoarthritis and MMPs gene polymorphisms). Rheumatology international. 2009; 29:383-388.

8. Young DA, Phillips BW, Lundy C, Nuttall RK, Hogan A, Schultz GA, Leco KJ, Clark IM and Edwards DR. Identification of an initiator-like element essential for the expression of the tissue inhibitor of metalloproteinases-4 (Timp-4) gene. Biochem J. 2002; 364:89-99.

9. Prieto-Alhambra D, Judge A, Javaid MK, Cooper C, Diez-Perez A and Arden NK. Incidence and risk factors for clinically diagnosed knee, hip and hand osteoarthritis: influences of age, gender and osteoarthritis affecting other joints. Annals of the rheumatic diseases. 2013:annrheumdis-2013-203355.
10. Lohmander LS, Englund PM, Dahl LL and Roos EM. The long-term consequence of anterior cruciate ligament and meniscus injuries osteoarthritis. The American journal of sports medicine. 2007; 35:1756-1769.

11. Zhang Y and Jordan JM. Epidemiology of Osteoarthritis. Clinics in Geriatric Medicine. 2010; 26:355-369.

12. Valdes AM and Spector TD. Genetic epidemiology of hip and knee osteoarthritis. Nature reviews Rheumatology. 2011; 7:23-32.

13. Pan F, Tian J, Winzenberg $\mathrm{T}$, Ding $\mathrm{C}$ and Jones $\mathrm{G}$. Association between GDF5 rs143383 polymorphism and knee osteoarthritis: an updated meta-analysis based on 23,995 subjects. BMC musculoskeletal disorders. 2014; 15:404.

14. Kerkhof HJ, Lories RJ, Meulenbelt I, Jonsdottir I, Valdes AM, Arp P, Ingvarsson T, Jhamai M, Jonsson H, Stolk L, Thorleifsson G, Zhai G, Zhang F, Zhu Y, van der Breggen $\mathrm{R}$, Carr A, et al. A genome-wide association study identifies an osteoarthritis susceptibility locus on chromosome 7q22. Arthritis and rheumatism. 2010; 62:499-510.

15. Spector TD and MacGregor AJ. Risk factors for osteoarthritis: genetics11Supported by Procter \& Gamble Pharmaceuticals, Mason, OH. Osteoarthritis and Cartilage. 2004; 12:39-44.

16. Keam B-S, Hwang J-Y, Go M-J, Heo J-Y, Park M-S, Lee J-Y, Kim N-H, Park M, Oh J-H, Kim D-H, Jeong J-Y, Lee J-Y, Han B-G and Lee J-Y. Genome-wide Association Study Identified TIMP2 Genetic Variant with Susceptibility to Osteoarthritis. Genomics \& Informatics. 2011; 9:121-126.

17. Ji JB, Li XF, Liu L, Wang GZ and Yan XF. Effect of low intensity pulsed ultrasound on expression of TIMP-2 in serum and expression of mmp-13 in articular cartilage of rabbits with knee osteoarthritis. Asian Pacific journal of tropical medicine. 2015; 8:1043-1048.

18. Zhang FJ, Yu WB, Luo W, Gao SG, Li YS and Lei GH. Effect of osteopontin on TIMP-1 and TIMP-2 mRNA in chondrocytes of human knee osteoarthritis in vitro. Experimental and therapeutic medicine. 2014; 8:391-394.

19. Lee HJ, Lee GH, Nah S, Lee KH, Yang H, Kim YM, Chun W, Hong S and Kim S. Association of TIMP-4 gene polymorphism with the risk of osteoarthritis in the Korean population. Rheumatology international. 2008; 28:845-850.

20. Pelletier JP, Mineau F, Faure MP and Martel-Pelletier J. Imbalance between the mechanisms of activation and inhibition of metalloproteases in the early lesions of experimental osteoarthritis. Arthritis \& Rheumatism. 1990; 33:1466-1476.

21. Knäuper V, López-Otin C, Smith B, Knight G and Murphy G. Biochemical characterization of human collagenase-3. Journal of Biological Chemistry. 1996; 271:1544-1550.

22. Tetlow LC, Adlam DJ and Woolley DE. Matrix metalloproteinase and proinflammatory cytokine production by chondrocytes of human osteoarthritic cartilage: associations with degenerative changes. Arthritis \& Rheumatism. 2001; 44:585-594. 
23. Chen X, Guo Z, Wang P and Xu M. Erythropoietin Modulates Imbalance of Matrix Metalloproteinase-2 and Tissue Inhibitor of Metalloproteinase-2 in Doxorubicin-induced Cardiotoxicity. Heart, Lung and Circulation. 2014; 23:772-777.

24. Rodriguez-Perez JM, Martinez-Rodriguez N, VargasAlarcon G, Vallejo M, Monroy-Munoz IE, Posadas-Romero C, Kimura-Hayama E, Juarez-Cedillo T, Fragoso JM and Perez-Hernandez N. TIMP2 gene polymorphisms are associated with hypertension in patients with myocardial infarction. Journal of genetics. 2014; 93:517-522.

25. El Khoury L, Ribbans WJ and Raleigh SM. MMP3 and TIMP2 gene variants as predisposing factors for Achilles tendon pathologies: Attempted replication study in a British case-control cohort. Meta gene. 2016; 9:52-55.

26. Zhang S, Gao X, Yang J and Ji Z. TIMP-2 G-418C polymorphism and cancer risk: A meta-analysis. Journal of cancer research and therapeutics. 2015; 11:308-312.

27. Kunt AT, Isbir S, Gormus U, Kahraman OT, Arsan S, Yilmaz SG and Isbir T. Polymorphisms of MMP9 and TIMP2 in Patients with Varicose Veins. In vivo (Athens, Greece). 2015; 29:461-465.

28. Blanco FJ, Moller I, Romera M, Rozadilla A, SanchezLazaro JA, Rodriguez A, Galvez J, Fores J, Monfort J, Ojeda S, Moragues C, Caracuel MA, Clavaguera T, Valdes C, Soler JM, Orellana C, et al. Improved prediction of knee osteoarthritis progression by genetic polymorphisms: the Arthrotest Study. Rheumatology (Oxford, England). 2015; 54:1236-1243.

29. Peterson NB, Beeghly-Fadiel A, Gao YT, Long J, Cai $\mathrm{Q}$, Shu XO and Zheng W. Polymorphisms in tissue inhibitors of metalloproteinases- 2 and -3 and breast cancer susceptibility and survival. Int J Cancer. 2009; 125:844-850.

30. Arden N and Nevitt MC. Osteoarthritis: epidemiology. Best practice \& research Clinical rheumatology. 2006; 20:3-25.

31. Sowers MF, Hochberg M, Crabbe JP, Muhich A, Crutchfield $\mathrm{M}$ and Updike S. Association of bone mineral density and sex hormone levels with osteoarthritis of the hand and knee in premenopausal women. American journal of epidemiology. 1996; 143:38-47.

32. Cauley JA, Kwoh CK, Egeland G, Nevitt MC, Cooperstein L, Rohay J, Towers A and Gutai JP. Serum sex hormones and severity of osteoarthritis of the hand. The Journal of rheumatology. 1993; 20:1170-1175.

33. Shin MH, Lee SJ, Kee SJ, Song SK, Kweon SS, Park DJ, Park YW, Lee SS and Kim TJ. Genetic association analysis of GDF5 and ADAM12 for knee osteoarthritis. Joint, bone, spine : revue du rhumatisme. 2012; 79:488-491.

34. Vaes RB, Rivadeneira F, Kerkhof JM, Hofman A, Pols HA, Uitterlinden AG and van Meurs JB. Genetic variation in the GDF5 region is associated with osteoarthritis, height, hip axis length and fracture risk: the Rotterdam study. Ann Rheum Dis. 2009; 68:1754-1760.

35. Evangelou E, Chapman K, Meulenbelt I, Karassa FB, Loughlin J, Carr A, Doherty M, Doherty S, Gomez-Reino JJ, Gonzalez A, Halldorsson BV, Hauksson VB, Hofman A, Hart DJ, Ikegawa S, Ingvarsson T, et al. Large-scale analysis of association between GDF5 and FRZB variants and osteoarthritis of the hip, knee, and hand. Arthritis and rheumatism. 2009; 60:1710-1721.

36. Waimann CA, Fernandez-Mazarambroz RJ, Cantor SB, Lopez-Olivo MA, Barbo AG, Landon GC, Siff SJ, Lin H and Suarez-Almazor ME. Effect of Body Mass Index and Psychosocial Traits on Total Knee Replacement Costs in Patients with Osteoarthritis. The Journal of rheumatology. 2016:jrheum. 151301.

37. Pereira D, Severo M, Ramos E, Branco J, Santos RA, Costa L, Lucas R and Barros H. Potential role of age, sex, body mass index and pain to identify patients with knee osteoarthritis. International journal of rheumatic diseases. 2015.

38. Ashford S and Williard J. Osteoarthritis: A review. The Nurse Practitioner. 2014; 39:1-8.

39. Gabriel S, Ziaugra L and Tabbaa D. SNP genotyping using the Sequenom MassARRAY iPLEX platform. Current protocols in human genetics. 2009:2.12. 11-12.12. 16.

40. Checa M, Ruiz V, Montano M, Velazquez-Cruz R, Selman $\mathrm{M}$ and Pardo A. MMP-1 polymorphisms and the risk of idiopathic pulmonary fibrosis. Human genetics. 2008; 124:465-472.

41. Yunus Z, Liu L, Wang H, Zhang L, Li X, Geng T, Kang L, Jin T and Chen C. Genetic polymorphisms of pharmacogenomic VIP variants in the Kyrgyz population from northwest China. Gene. 2013; 529:88-93.

42. Speedy HE, Di Bernardo MC, Sava GP, Dyer MJ, Holroyd A, Wang Y, Sunter NJ, Mansouri L, Juliusson G and Smedby KE. A genome-wide association study identifies multiple susceptibility loci for chronic lymphocytic leukemia. Nature genetics. 2014; 46:56-60.

43. Bland JM and Altman DG. The odds ratio. Bmj. 2000; 320:1468. 